PROCEEDINGS OF THE

AMERICAN MATHEMATICAL SOCIETY

Volume 132, Number 7, Pages 2095-2101

S 0002-9939(04)07403-9

Article electronically published on February 6, 2004

\title{
THE DUAL SPECTRAL SET CONJECTURE
}

\author{
STEEN PEDERSEN
}

(Communicated by David R. Larson)

\begin{abstract}
Suppose that $\Lambda=(a \mathbf{Z}+b) \cup(c \mathbf{Z}+d)$ where $a, b, c, d$ are real numbers such that $a \neq 0$ and $c \neq 0$. The union is not assumed to be disjoint. It is shown that the translates $\Omega+\lambda, \lambda \in \Lambda$, tile the real line for some bounded measurable set $\Omega$ if and only if the exponentials $e_{\lambda}(x)=e^{i 2 \pi \lambda x}, \lambda \in \Lambda$, form an orthogonal basis for some bounded measurable set $\Omega^{\prime}$.
\end{abstract}

\section{INTRODUCTION}

The relationship between tilings of $\mathbf{R}^{d}$ by translations of a single tile and spectral properties of the tile dates back to an old conjecture of Bent Fuglede. So far, there are few sharp results. Here we present a setting in $\mathbf{R}$ where the tiling property is equivalent to the spectral property suggested by Fuglede.

Definition 1.1. A domain is a bounded Lebesgue measurable set $\Omega \subset \mathbf{R}$ with Lebesgue measure $0<|\Omega|<\infty$.

A pair $(\Omega, \Lambda)$ consisting of a domain $\Omega$ and a set $\Lambda \subset \mathbf{R}$ is called a spectral pair if the exponentials $\mathcal{E}_{\Lambda}=\left\{e_{\lambda}: \lambda \in \Lambda\right\}$ form an orthogonal basis for $L^{2}(\Omega)$, where $e_{\lambda}(x)=e(\lambda x)=e^{2 \pi i \lambda x}$. A domain $\Omega$ is a spectral set if $(\Omega, \Lambda)$ is a spectral pair for some set $\Lambda$, and a set $\Lambda$ is a spectrum if $(\Omega, \Lambda)$ is a spectral pair for some domain $\Omega$. A pair $(\Omega, \Lambda)$ consisting of a domain $\Omega$ and a set $\Lambda \subset \mathbf{R}$ is called a tiling pair if $\bigcup_{\lambda \in \Lambda}(\Omega+\lambda)$ is a measure-theoretic partition of $\mathbf{R}$, i.e., if $\sum_{\lambda \in \Lambda} \chi_{\Omega+\lambda}(x)=1$ for a.e. $x \in \mathbf{R}$. Here $\Omega+\lambda=\{x+\lambda: x \in \Omega\}$ and $\chi_{T}(x)$ is the characteristic function of the set $T$. A domain $\Omega$ is a tile if $(\Omega, \Lambda)$ is a tiling pair for some $\Lambda$, and a set $\Lambda$ is a tiling set if $(\Omega, \Lambda)$ is a tiling pair for some domain $\Omega$.

Conjecture 1.2 (The spectral set conjecture [Fug74]). A domain is a spectral set if and only if it is a tile.

Conjecture 1.3 (The dual spectral set conjecture [JP99]). A subset of $\mathbf{R}$ is a spectrum if and only if it is a tiling set.

It follows from results in [JP99] that suitable generalizations of these conjectures, where both $\Omega$ and $\Lambda$ are replaced by positive measures, are equivalent.

Received by the editors April 15, 2003.

2000 Mathematics Subject Classification. Primary 42A99, 42C99, 51M04, 52C99.

Key words and phrases. Fourier basis, non-harmonic Fourier series, tiling, spectral set, spectral pair.

(C)2004 American Mathematical Society 
The spectral set conjecture has been studied in numerous recent papers, for example, [KT01], [JP99], [Kol00, [Lab01], [LS01], [LW97], [Ped96, [PW01] and the references therein.

Both the tiling pair and the spectral pair condition can be viewed as a tiling condition on a certain function associated with $\Omega$. A pair $(\Omega, \Lambda)$ is a tiling pair iff

$$
\sum_{\lambda \in \Lambda} \chi_{\Omega}(x-\lambda)=1 \text {, a.e. } x \in \mathbf{R},
$$

whereas $(\Omega, \Lambda)$ is a spectral pair iff

$$
\sum_{\lambda \in \Lambda}|\widehat{\chi \Omega}(x-\lambda)|^{2}=|\Omega|^{2} \text {, a.e. } x \in \mathbf{R} .
$$

Here $\widehat{\chi \Omega}$ is the Fourier transform

$$
\widehat{\chi \Omega}(\xi)=\int_{\Omega} e_{\xi}(-x) d x
$$

of the characteristic function $\chi_{\Omega}$ of the domain $\Omega$.

Incidentally, this shows that tiling questions are "easier" than the corresponding spectral questions, since the tiling questions involve tiling by translates of a $\{0,1\}$ valued function while spectral questions involve tilings by translates of a continuous function whose values include 0 and $|\widehat{\chi \Omega}(0)|^{2}=|\Omega|^{2}$.

It is known Fug74 that the only spectra for an interval of length $\ell$ are the translates of the lattice $\frac{1}{\ell} \mathbf{Z}$. The union of two translated lattices is a set of the form

$$
\Lambda=(a+b \mathbf{Z}) \cup(c+d \mathbf{Z}),
$$

where $a, b, c, d$ are real numbers, $b \neq 0$ and $d \neq 0$. Here $a \mathbf{Z}+b=\{a n+b: n \in \mathbf{Z}\}$.

Theorem 1.4. Let $\Lambda \subset \mathbf{R}$ be the union of two translated lattices. Then $(\Omega, \Lambda)$ is the spectral pair for some domain $\Omega$ if and only if $\left(\Omega^{\prime}, \Lambda\right)$ is a tiling pair for some domain $\Omega^{\prime}$.

I will prove this result below, thereby establishing the dual spectral set conjecture for sets that are the union of two translated lattices. It is not assumed that the union of the two translated lattices is disjoint.

Assuming that the spectrum is the union of two translated lattices does not imply that a corresponding spectral set must be a union of two intervals. More explicitly, there are sets with spectrum $\Lambda=2 \mathbf{Z} \cup\left(2 \mathbf{Z}+\frac{1}{2}\right)$ that are not the union of two intervals; for example, $\Omega=\left(0, \frac{1}{2}\right) \cup\left(1, \frac{5}{4}\right) \cup\left(\frac{13}{4}, \frac{7}{2}\right)$ is a simple example of such a set. Similarly, one can construct spectral sets with infinitely many connected components and spectrum $2 \mathbf{Z} \cup\left(2 \mathbf{Z}+\frac{1}{2}\right)$.

The following simple lemma is well known.

Lemma 1.5 (Affine invariance). Let $\Lambda \subset \mathbf{R}$, let $\Omega$ be a domain, and let $a, b, c \in \mathbf{R}$. Suppose $a \neq 0$.

(1) $(\Omega, \Lambda)$ is a tiling pair if and only if $(a \Omega+b, a \Lambda+c)$ is a tiling pair.

(2) $(\Omega, \Lambda)$ is a spectral pair if and only if $\left(a \Omega+b, \frac{1}{a} \Lambda+c\right)$ is a spectral pair.

By affine invariance of the problem under consideration it is sufficient to consider the case where one of the lattices is of a convenient form. Below we will assume $\Lambda=\mathbf{Z} \cup(a \mathbf{Z}+b)$ for some $a, b \in \mathbf{R}$ with $0 \leq b<a \leq 1$.

In Sections 2 and $[3$ we establish Theorem 1.4 


\section{ANy SPECTRUM is A TILING SET}

Suppose $\Lambda$ is a spectrum. We will show that $\Lambda$ is a tiling set by constructing a tile $\Omega^{\prime}$ such that $\left(\Omega^{\prime}, \Lambda\right)$ is a tiling pair. Let $\Omega$ be a domain such that $(\Omega, \Lambda)$ is a spectral pair.

\subsection{The nonzero constant $a$ is rational.}

Proof. The inner product between two exponentials $e_{\lambda}$ and $e_{\lambda^{\prime}}$ is

$$
\left\langle e_{\lambda} \mid e_{\lambda^{\prime}}\right\rangle_{\Omega}=\widehat{\chi \Omega}\left(\lambda-\lambda^{\prime}\right) .
$$

It follows that the two exponentials $e_{\lambda}$ and $e_{\lambda^{\prime}}$ are orthogonal exactly when $\widehat{\chi \Omega}\left(\lambda-\lambda^{\prime}\right)=0$. In particular, if the exponentials $\mathcal{E}_{\lambda}$ are orthogonal, then the set of non-zero differences $(\Lambda-\Lambda) \backslash\{0\}$ is a subset of the set of zeros of the function $\widehat{\chi}_{\Omega}$. The function $\widehat{\chi_{\Omega}}: \mathbf{R} \rightarrow \mathbf{C}$ is continuous, and $\widehat{\chi_{\Omega}}(0)=|\Omega| \neq 0$. Hence there exists $\varepsilon>0$ so that $\widehat{\chi \Omega}(\xi) \neq 0$ for any $|\xi|<\varepsilon$.

Suppose $a$ is irrational. Let $\Lambda^{\prime}=\{k+a l-b: k, l \in \mathbf{Z}\}$. Since $a$ is irrational, the set $\{k+a l: k, l \in \mathbf{Z}\}$ is dense in $\mathbf{R}$; hence there is a point $x$ in $\Lambda^{\prime}$ with $0<|x|<\varepsilon$. Since $\Lambda^{\prime} \subseteq \Lambda-\Lambda$ and $x \neq 0$, it follows from orthogonality that $\widehat{\chi_{\Omega}}(x)=0$. On the other hand, it follows from $|x|<\varepsilon$ that $\widehat{\chi_{\Omega}}(x) \neq 0$. This contradiction shows that $a$ is rational.

It is a consequence of this proof that $\Lambda$ is uniformly discrete in the sense that if $\lambda \neq \lambda^{\prime}$ are in $\Lambda$, then $\left|\lambda-\lambda^{\prime}\right| \geq \varepsilon$.

Up to a change of scale this allows us to assume that any spectrum is of the form $\Lambda=\mathbf{Z}+B$ for some finite set $B$. However, we need more precise information.

Since $a \in \mathbf{Q}$, we have $a=\frac{q}{p}$ for some positive integers $p, q$ with greatest common divisor $\operatorname{gcd}(p, q)=1$. It follows that $p \Lambda=p \mathbf{Z} \cup(q \mathbf{Z}+r)$, where $r=p b \in \mathbf{R}$ and $0 \leq r<q$.

2.2. Suppose $r \in \mathbf{Z}$. Since $\operatorname{gcd}(p, q)=1$, it follows that $p \Lambda-p \Lambda \supseteq\{p m+q n+r$ : $m, n \in \mathbf{Z}\}=\mathbf{Z}$. Therefore the exponentials $\mathcal{E}_{\mathbf{Z}}$ are orthogonal in $L^{2}\left(\frac{1}{p} \Omega\right)$. Now $\mathcal{E}_{p \Lambda} \subseteq \mathcal{E}_{\mathbf{Z}}$ and $\mathcal{E}_{p \Lambda}$ is a maximal orthogonal subset of $L^{2}\left(\frac{1}{p} \Omega\right)$; it follows that $\varepsilon_{p \Lambda}=\varepsilon_{\mathbf{Z}}$. Consequently, $p \Lambda=\mathbf{Z}$. Since $([0,1), \mathbf{Z})$ is a tiling pair, we may use $\Omega^{\prime}=[0, p)$.

Remark 2.1. In light of what follows it is perhaps interesting to note that the only ways $p \mathbf{Z} \cup(r+q \mathbf{Z})$ can equal $\mathbf{Z}$ for integers $p \in \mathbf{N}, q \in \mathbf{N}$ and $r \in \mathbf{N}_{0}$ are $(i)$ $p=q=1$ and $($ ii) $p=q=2$ and $r$ is odd. The second possibility is ruled out by $\operatorname{gcd}(p, q)=1$.

2.3. Suppose $r \notin \mathbf{Z}$. Since $p \mathbf{Z}$ is a subset of $p \Lambda$, the exponentials $\mathcal{E}_{p \mathbf{Z}}$ are orthogonal. It follows from Theorem 6.2 in JJP92 that $p^{\prime}:=p\left|\frac{1}{p} \Omega\right|$ is an integer. Similarly, the exponentials $\mathcal{E}_{q \mathbf{Z}}$ are orthogonal, and therefore $q^{\prime}:=q\left|\frac{1}{p} \Omega\right|$ is an integer.

Now $\frac{p^{\prime}}{p}=\left|\frac{1}{p} \Omega\right|=\frac{q^{\prime}}{q}$. In particular, $p^{\prime} q=p q^{\prime}$. Since $\operatorname{gcd}(p, q)=1$, it follows that $p$ divides $p^{\prime}$ and $q$ divides $q^{\prime}$. Thus $\left|\frac{1}{p} \Omega\right|$ is an integer. 
If $(\widetilde{\Omega}, \widetilde{\Lambda})$ is a spectral pair, then it follows from (1.2) and Lemma 2.3(ii) in KL96 that the asymptotic density

$$
\operatorname{dens}(\widetilde{\Lambda})=\lim _{R \rightarrow \infty} \frac{\#(\widetilde{\Lambda} \cap[-R, R])}{2 R}
$$

exists and equals $|\widetilde{\Omega}|$.

Clearly, $\operatorname{dens}(p \mathbf{Z})=\frac{1}{p}$ and $\operatorname{dens}(q \mathbf{Z}+r)=\frac{1}{q}$. Using disjointness of $p \mathbf{Z}$ and $q \mathbf{Z}+r$, we get dens $(p \mathbf{Z} \cup(q \mathbf{Z}+r))=\operatorname{dens}(p \mathbf{Z})+\operatorname{dens}(q \mathbf{Z}+r)$. Thus

$$
\left|\frac{1}{p} \Omega\right|=\frac{1}{p}+\frac{1}{q} .
$$

Since $p, q,\left|\frac{1}{p} \Omega\right|$ all are positive integers, it follows that either $(i): p=q=2$, $\left|\frac{1}{p} \Omega\right|=1$, and $r \notin 2 \mathbf{Z}$, or $(i i): p=q=1$ and $\left|\frac{1}{p} \Omega\right|=2$ if $r \notin \mathbf{Z}$ and $\left|\frac{1}{p} \Omega\right|=1$ if $r \in \mathbf{Z}$. Since $\operatorname{gcd}(p, q)=1$, case $(i)$ does not occur. We are left to consider $p \Lambda=\mathbf{Z} \cup(\mathbf{Z}+r)$ with $0<r<1$, but this allows us to use [JP87]. In fact, the proof of Theorem 5.1 in [JP87] shows that there exists an integer $n$ such that $0<\left|\frac{1}{p} \Omega \cap\left(n+\frac{1}{p} \Omega\right)\right|<\left|\frac{1}{p} \Omega\right|$. Also, if $n$ is any such integer, then $e^{i 2 \pi n r}=-1$. It follows that

$$
r=\frac{m}{2 n}
$$

for some odd integer $m$. Hence

$$
p \Lambda=\mathbf{Z} \cup\left(\mathbf{Z}+\frac{m}{2 n}\right),
$$

where $n \neq 0$ is an integer and $m$ is an odd integer. But the set in (2.2) is a tiling set for

$$
\frac{1}{p} \Omega^{\prime}=\left[0, \frac{1}{2 n}\right)+\frac{1}{n}\{0,1, \ldots, n-1\} .
$$

Remark 2.2. In connection with the form of (2.2), note that $\Lambda=\mathbf{Z} \cup\left(\mathbf{Z}+\frac{1}{3}\right)$ is not a tiling set for any tile. This is easily verified directly.

\section{Any tiling Set is a SPECTRUm}

Suppose $\Lambda=\mathbf{Z} \cup(a \mathbf{Z}+b)$ is a tiling set. We will construct a domain $\Omega^{\prime}$ such that $\left(\Omega^{\prime}, \Lambda\right)$ is a spectral pair. Let $\Omega$ be a domain such that $(\Omega, \Lambda)$ is a tiling pair. We may assume that $0 \leq b<a \leq 1$.

It follows from Theorem 1.1 in KL96 that $a$ is a rational number. Hence, we may write $a=\frac{q}{p}$, where $p, q \in \mathbf{N}$ and $\operatorname{gcd}(p, q)=1$. Multiplying by $p$, we have that $p \Lambda=p \mathbf{Z} \cup(q \mathbf{Z}+r)$ is a tiling set for $p \Omega$; here $r=p b$ as above. Note that $0 \leq r<q \leq p$.

It follows from (1.1) and Lemma 2.3 in [KL96] that

$$
|\widetilde{\Omega}|=\frac{1}{\operatorname{dens}(\widetilde{\Lambda})}
$$

for every tiling pair $(\widetilde{\Omega}, \widetilde{\Lambda})$. This replaces $(2.1)$ in the proof of the converse. 
3.1. Suppose $r \in \mathbf{Z}$. By the tiling assumption, $|(p \Omega+p m) \cap(p \Omega+q n+r)|=0$ for all integers $m, n$ for which $p m \neq r+q n$. Subtracting $p m$, we have $\mid p \Omega \cap(p \Omega+q n+$ $r-p m) \mid=0$ for all integers $m, n$ for which $r+q n-p m \neq 0$. Since $\operatorname{gcd}(p, q)=1$, we conclude that $|p \Omega \cap(p \Omega+k)|=0$ for all integers $k \neq 0$. It follows that

$$
|p \Omega| \leq 1 .
$$

Now

$$
\operatorname{dens}(p \mathbf{Z} \cup(q \mathbf{Z}+r)) \leq \operatorname{dens}(p \mathbf{Z})+\operatorname{dens}(q \mathbf{Z}+r)=\frac{1}{p}+\frac{1}{q} .
$$

It follows from (3.2) and (3.1) that

$$
1 \geq|p \Omega|=\frac{1}{\operatorname{dens}(p \Lambda)} \geq \frac{p q}{p+q} .
$$

The only positive integers $p, q$ solving $p q \leq p+q$ are $(i) p=q=1$, (ii) $p=2, q=1$, (iii) $p=1, q=2$, and (iv) $p=q=2$. In our situation $q \leq p$ and $\operatorname{gcd}(p, q)=1$. So only (i) and (ii) are operative. In case $(i), \Lambda=\mathbf{Z}$. In case $(i i), 0 \leq r<q$ and $r \in \mathbf{Z}$ imply $r=0$. Again we have $\Lambda=\mathbf{Z}$. Hence, in both cases, $\left(\Omega^{\prime}, \Lambda\right)$ is a spectral pair if $\Omega^{\prime}=[0,1)$.

3.2. Suppose $r \notin \mathbf{Z}$. We begin by showing that $p=q=1$.

Proof that $p=q=1$. Using arguments similar to those we used in Subsection 3.1. we have

$$
|(p \Omega+m+r) \cap(p \Omega+n)|=0
$$

for all integers $m, n$, and we have the equality

$$
|p \Omega|=\frac{p q}{p+q}
$$

since $p \mathbf{Z} \cap(q \mathbf{Z}+r)=\emptyset$ implies $\operatorname{dens}(p \mathbf{Z} \cup(q \mathbf{Z}+r))=\operatorname{dens}(p \mathbf{Z})+\operatorname{dens}(q \mathbf{Z}+r)$. It follows from (3.3) that

$$
|(p \Omega+\mathbf{Z}) \cap(p \Omega+\mathbf{Z}+r)|=0 .
$$

But $p \Omega+p \mathbf{Z} \subseteq p \Omega+\mathbf{Z}, p \Omega+q \mathbf{Z}+r \subseteq p \Omega+\mathbf{Z}+r$, and $(p \Omega+p \mathbf{Z}) \cup(p \Omega+q \mathbf{Z}+r)=\mathbf{R}$, up to a set of measure zero. It follows that $p \Omega+\mathbf{Z}=p \Omega+p \mathbf{Z}$ and $p \Omega+\mathbf{Z}+r=$ $p \Omega+q \mathbf{Z}+r$, both up to sets of measure zero. Since $p \Omega+\mathbf{Z}=(p \Omega+\mathbf{Z}+r)-r$, we have

$$
p \Omega+p \mathbf{Z}=p \Omega+q \mathbf{Z}
$$

up to sets of measure zero. It follows that $p \mathbf{Z} \cup(p \mathbf{Z}+r)$ and $q \mathbf{Z} \cup(q \mathbf{Z}+r)$ both are tiling sets corresponding to the tile $p \Omega$. Using arguments similar to the one leading to (3.4) on each of these tiling sets, we have $|p \Omega|=\frac{p}{2}$ and $|p \Omega|=\frac{q}{2}$, hence $p=q$. Using $\operatorname{gcd}(p, q)=1$, we conclude that $p=q=1$.

Hence we have shown that $\Lambda=\mathbf{Z} \cup(\mathbf{Z}+r)$ for some $0<r<1$. Now we want to show that $r$ is rational.

Proof that $r$ is rational. This proof borrows from the proof of Theorem 2 in [LW96]. Suppose $r \notin \mathbf{Q}$. By (3.4) we have

$$
|\Omega|=\frac{1}{2} .
$$

Let $U=\Omega \cup(\Omega+r)$; then $(U, \mathbf{Z})$ is a tiling pair and

$$
\begin{aligned}
\widehat{\chi_{U}}(\xi) & =\int_{\Omega} e(t \xi) d t+\int_{\Omega+r} e(t \xi) d t \\
& =(1+e(r \xi)) \widehat{\chi \Omega}(\xi) .
\end{aligned}
$$


If $n \in \mathbf{Z}$ is a nonzero integer, then $1+e(r n) \neq 0$, since $r \notin \mathbf{Q}$. Furthermore,

$$
0=\widehat{\chi U}(n)=(1+e(r n)) \widehat{\chi \Omega}(n) .
$$

It follows that $\widehat{\chi \Omega}(n)=0$. Hence the exponentials $\mathcal{E}_{\mathbf{Z}}$ form an orthogonal system in $L^{2}(\Omega)$. It follows from Theorem 6.2 in [JP92 that $|\Omega|$ is an integer. This contradicts (3.5). Hence $r$ is rational.

Completion of the proof of Theorem 1.4 Hence $\Omega$ is a tile with tiling set

$$
\Lambda=\mathbf{Z} \cup\left(\mathbf{Z}+\frac{s}{t}\right)=\mathbf{Z}+\left\{0, \frac{s}{t}\right\},
$$

where $0<s<t$ are integers and $\operatorname{gcd}(s, t)=1$. Let $a_{1}=0$ and $a_{2}=s$. By Theorem 3 in [LW96, equation (3.6) implies that there exist an integer $K \geq 1$ and integers $\left(b_{k}\right)_{k=1}^{K}$ such that $0 \leq b_{k}<t$, and such that every integer $0 \leq j<t$ can be written (modulo $t$ ) uniquely as $j=a_{n}+b_{k}$ with $1 \leq n \leq 2$ and $1 \leq k \leq K$. It follows that $t=2 K$; in particular, $t$ is an even integer. Since $\operatorname{gcd}(s, t)=1$, we conclude that $s$ is an odd integer. Thus $\Lambda=\mathbf{Z} \cup\left(\mathbf{Z}+\frac{2 n-1}{2 K}\right)$ for some integers $K \neq 0$ and $n$. Let $\Omega^{\prime}=[0,1) \cup[K, K+1$ ). By [JP87] (the result is a special case of Theorem 2.1 in [JP94] $)$ it follows that $\left(\Omega^{\prime}, \Lambda\right)$ is a spectral pair. The important fact is that $e\left(K \frac{s}{t}\right)=-1$.

\section{Must the domains Be Bounded?}

In Fug74 Fuglede stated the Spectral Set Conjecture for Lebesgue measurable sets $\Omega$ with $0<|\Omega|<\infty$. In particular, he did not assume that $\Omega$ is bounded.

Thus we wish to replace Definition 1.1 by

Definition 4.1. A weak domain is a Lebesgue measurable set $\Omega \subset \mathbf{R}$ with Lebesgue measure $0<|\Omega|<\infty$.

Examining the proof of Theorem 1.4 above, we see that we can replace "domain" by "weak domain" almost everywhere. The only thing that does not work is the appeal to Theorem 1.1 in KL96 at the beginning of Section 3. If we only consider $\Lambda=\mathbf{Z} \cup(a \mathbf{Z}+b)$ with $\mathbf{Z} \cap(a \mathbf{Z}+b)=\emptyset$, then we can use the argument from Subsection 3.2 (without knowing that $a$ is rational) to show that $a=1$. Thus we have the following version of Theorem 1.4 .

Theorem 4.2. Let $\Lambda=\mathbf{Z} \cup(a \mathbf{Z}+b)$ be such that $\mathbf{Z} \cap(a \mathbf{Z}+b)=\emptyset$. Then $(\Omega, \Lambda)$ is the spectral pair for some weak domain $\Omega$ if and only if $\left(\Omega^{\prime}, \Lambda\right)$ is a tiling pair for some weak domain $\Omega^{\prime}$.

Thus, using our approach to this particular version of the Dual Spectral Set Conjecture, we are able to trade boundedness of $\Omega$ for disjointness of the lattices in $\Lambda$.

The definitions of spectral pair and of tiling pair make sense in $\mathbf{R}^{d}$. In this paper we only considered the case $d=1$. It is natural to wonder if one could prove an analogue of Theorem 1.4 in $\mathbf{R}^{d}$ with $d \geq 2$.

\section{ACKNOWLEDGMENT}

The author thanks Palle E. T. Jorgensen for helpful comments on a preliminary version of this paper. 


\section{REFERENCES}

[Fug74] Bent Fuglede, Commuting self-adjoint partial differential operators and a group theoretic problem, J. Funct. Anal. 16 (1974), 101-121. MR 57:10500

[IKT01] Alex Iosevich, Nets Katz, and Terrence Tao, Convex bodies with a point of curvature do not have Fourier bases, Amer. J. Math. 123 (2001), 115-120. MR 2002g:42011

[JP87] Palle E. T. Jorgensen and Steen Pedersen, Harmonic analysis on tori, Acta Appl. Math. 10 (1987), 87-99. MR 89e:22010

[JP92] Palle E. T. Jorgensen and Steen Pedersen, Spectral theory for Borel sets in $R^{n}$ of finite measure, J. Funct. Anal. 107 (1992), 74-104. MR 93k:47005

[JP94] Palle E. T. Jorgensen and Steen Pedersen, Harmonic analysis and fractal limit-measures induced by representations of a certain $C^{*}$-algebra, J. Funct. Anal. 125 (1994), 90-110. MR 95i:47067

[JP99] Palle E. T. Jorgensen and Steen Pedersen, Spectral pairs in Cartesian coordinates, J. Fourier Anal. Appl. 5 (1999), 285-302. MR 2002d:42027

[KL96] Mihail N. Kolountzakis and Jeffrey C. Lagarias, Structure of tilings of the line by a function, Duke Math. J. 82 (1996), 653-678. MR 97d:11124

[Kol00] Mihail N. Kolountzakis, Packing, tiling, orthogonality and completeness, Bull. London Math. Soc. 32 (2000), 589-599. MR 2001g:52030

[Łab01] Izabella Łaba, Fuglede's conjecture for a union of two intervals, Proc. Amer. Math. Soc. 129 (2001), 2965-2972. MR 2002d:42007

[LS01] Jeffrey C. Lagarias and Sándor Szabó, Universal spectra and Tijdeman's conjecture on factorization of cyclic groups, J. Fourier Anal. Appl. 7 (2001), 63-70. MR 2002e:11107

[LW96] Jeffrey C. Lagarias and Yang Wang, Tiling the line with translates of one tile, Invent. Math. 124 (1996), 341-365. MR 96i:05040

[LW97] Jeffrey C. Lagarias and Yang Wang, Spectral sets and factorizations of finite abelian groups, J. Funct. Anal. 145 (1997), 73-98. MR 98b:47011b

[Ped96] Steen Pedersen, Spectral sets whose spectrum is a lattice with a base, J. Funct. Anal. 141 (1996), 496-509. MR 98b:47011a

[PW01] Steen Pedersen and Yang Wang, Universal spectra, universal tiling sets and the spectral set conjecture, Math. Scand. 88 (2001), 246-256. MR 2002k:52030

Department of Mathematics, Wright State University, Dayton, Ohio 45435

E-mail address: steen@math.wright.edu

$U R L:$ http://www.wright.edu/ steen.pedersen/ 